\title{
Socio-cultural Beliefs and Practices Influencing Institutional Delivery Service Utilization in Three Communities of Ethiopia: A Qualitative Study
}

\author{
Sabit Ababor ${ }^{1 *}$, Zewdie Birhanu ${ }^{2}$, Atkure Defar ${ }^{1}$, Kasahun Amenu ${ }^{1}$, Amanuel \\ Dibaba $^{1}$, Desalegn Araraso ${ }^{1}$,Yosef Gebreyohanes ${ }^{1}$, Mamuye Hadis ${ }^{1}$
}

OPEN ACCESS

Citation: Sabit Ababor, Zewdie Birhanu, Atkure Defar, Kasahun Amenu, Amanuel Dibaba, Desalegn Araraso, Yosef Gebreyohanes, Mamuye Hadis. Sociocultural Beliefs and Practices Influencing Institutional Delivery Service Utilization in Three Communities of Ethiopia: A Qualitative Study. Ethiop J Health Sci. 2019;29(3):343.

doi:http://dx.doi.org/10.4314/ ejhs.v29i3.6

Received: April 1, 2019

Accepted: April 4, 2019

Published: May 1, 2019

Copyright: () Sabit Ababor, et al. This is an open-access article distributed under the terms of the Creative Commons Attribution License, which permits unrestricted use, distribution, and reproduction in any medium, provided the original author and source are credited.

Funding: Ethiopian Public Health Institute

Competing Interests: The authors declare that this manuscript was approved by all authors in its form and that no competing interest exists.

Affiliation and Correspondence:

${ }^{1}$ Ethiopian Public Health Institute, Addis Ababa, Ethiopia

University ${ }^{2}$ Department of Health, Behavior, and Society, Faculty of Public Health, Jimma University, Jimma, Ethiopia

*Email:Sabitababor32@gmail.com

\author{
ABSTRACT
}

BACKGROUND: The influence of socio-cultural factors on institutional birth is not sufficiently documented in Ethiopia. Thus, this study explores socio-cultural beliefs and practices during childbirth and its influences on the utilization of institutional delivery services.

METHODS: A qualitative study was conducted in three regions of Ethiopia through eight focus group discussions (with women) and thirty in-depth interviews with key informants which included health workers, community volunteers, and leaders. The data were analyzed thematically.

RESULTS: The study identified six overarching socio-cultural factors influencing institutional birth in the study communities. The high preference for traditional birth attendants (TBAs) and home as it is intergenerational culture and suitable for privacy are among the factors. Correspondingly, culturally unacceptable birth practices at health facilities (such as birth position, physical assessment, delivery coach) and inconvenience of health facility setting to practice traditional birth rituals such as newborn welcoming ceremony made women avoid health facility birth. On the other hand, misperceptions and worries on medical interventions such as episiotomy, combined with mistreatment from health workers, and lack of parent engagement in delivery process discouraged women from seeking institutional birth. The provision of delivery service by male health workers was cited as a social taboo and against communities' belief system which prohibited women from giving birth at a health facility.

CONCLUSIONS: Multiple socio-cultural factors and perceptions were generally affected utilization of institutional birth in study communities. Hence, culturally competent interventions through education, re-orientation, and adaptation of beneficial norms combined with women friendly care are essential to promote health facility birth.

KEYWORDS: Ethiopia, Institutional delivery, Traditional birth attendants, Socio-cultural factors. 


\section{INTRODUCTION}

Despite tremendous progress in reduction of maternal death globally; it is still unacceptably high in many developing regions of the world (1). Approximately, 800 women die of preventable causes related to pregnancy and childbirth every day (1). Nearly all (99\%) of these deaths occur in developing countries (2). Despite substantial progress, maternal mortality in Ethiopia is among the highest in the world with 412 deaths per 100,000 live births (3). Unskilled birth attendance is one of the main causes of high maternal mortality in low-income countries (4).

Skilled care at birth is critical to reduce maternal death (5). It has been reported that a $10 \%$ increase in skilled birth attendance corresponds to $5 \%$ reduction in maternal deaths (6). Even though institutional delivery is a key strategy to reduce maternal deaths in Ethiopia, home delivery is still a common practice $(3,7)$. In Ethiopia, the proportion of deliveries attended at health facility was only $10 \%$ in 2011 and $26 \%$ in $2016(3,7)$. Health facility births are especially low in some regional states including Afar (6.8\%), Somali (7.6\%) and Benishangul-Gumuz $(9.1 \%)$ in 2011(3).

Socio-cultural beliefs, norms, values, and traditions have a major influence on decisionmaking whether a mother seeks skilled delivery care or not $(8,9)$. In countries like Ethiopia where cultural norms, values, and practices during childbirths are quite common and diverse, it is crucial to have a deeper understanding of sociocultural factors which possibly influence institutional birth. However, little is documented regarding socio-cultural beliefs and practices influencing utilization of institutional delivery service. This study, therefore, intended to explore socio-cultural values, norms, practices and perceptions during childbirth and how it influences the utilization of institutional delivery services. The findings from such studies could help inform policy and program planners for incorporating and providing a culture-sensitive delivery service.

\section{MATERIALS AND METHODS}

Study setting: The study was conducted in three selected regional states in Ethiopia: Afar, Somali, and Benishangul-Gumuz (BG). The institutional delivery service uptakes of the regions were $6.8 \%$ in Afar, $7.6 \%$ in Somali and $9.1 \%$ for BG in 2011 (7). From each regional state, a rural District having the lowest delivery service utilization was selected i.e. Dalifage in Afar, Dhagahbur in Somali and Asosa in BGBerta community. In 2013/2014, Dalifage District reported only1.6\% institutional delivery service utilization coverage while Asosa recorded 21\%. In the same period, the utilization coverage of Dhagahbur District was only $0.91 \%$.

Population and sample: A qualitative study with a grounded theory approach was conducted from September to October 2013. Study Districts were purposively selected in consultation with regional health bureaus considering areas with low utilization of institutional delivery services as the main criteria. From each selected District, one village was randomly selected. The data were collected through focus group discussions (FGDs) and key informant interviews (KIIs). Accordingly, a total of 8 FGDs (3 in Afar, 3 in BG and 2 in Somali) were conducted from women who gave birth in the last three years preceding the study time. Women were selected through purposive sampling approach to ensure diversity in age, marital status, parity, and education. In each FGD, the participants ranged from 8 to 10, and all were Muslims. Each FGD was conducted by two experts with rich experience in qualitative technique and fluent in local languages. Likewise, a total of 30 KIIs (10 in each region) were conducted with diverse groups of key informants such as health extension workers (HEWs), community leaders, traditional birth attendants (TBAs), midwives, social mobilizers, and relevant office representatives at District level. All discussions and interviews were recorded using a digital voice recorder and note taking. Recurrence of ideas determined the size of the interviewee and FGD.

Data collection tools and methods: Open-ended guides were used to collect the data. The guiding

DOI: http://dx.doi.org/10.4314/ejhs.v29i3.6 
questions were accompanied by several probes questions to get a deep insight into participants' beliefs. The guides were pretested to ensure their relevance and appropriateness. Data collectors were trained by senior researchers. Each FGD was conducted by two individuals who served as moderator and note-taker.

Data analysis: Data from FGDs and KIIs were transcribed verbatim and then translated into English language for analysis. Two researchers critically reviewed all the transcripts for accuracy and completeness. Each transcript was read and re-read by two investigators (SA and ZB). An initial coding structure was then developed through open coding method. In order to ensure the relevance and appropriateness of the coding structure, one coder (SA) conducted open coding of emergent themes associated with the study objectives, guided by grounded theory (10). The second coder (ZB) independently coded the same transcripts. Finally, the two coders and the research team reviewed the coded transcripts to reach consensus on coding structure and consistent code definitions which was used to code all transcripts. Results were organized into themes and sub-themes and supported with key quotes and narrations.

Trustworthiness: To ensure the quality of the data, data collectors were recruited from study communities and were trained well. The appropriateness and relevance of the guides were ensured through expert reviews and pre-testing. The field team held a debriefing session each day during the entire fieldwork. Facilitators' and note takers' impressions were documented for each data source. Data from FGDs and KIIs were triangulated to ensure credibility.

Ethical considerations: The Ethiopian Public Health Institute's Ethical Review Board approved the study, and permission was obtained from all relevant offices. Informed verbal consent was obtained from all participants.

\section{RESULTS}

Background characteristics of the participants: In 8 FGDs, 78 women with age ranged from fifteen to forty-nine participated. Except four, all of them were married. In terms of education, fifty-two of them had no formal education and the remaining attended primary and secondary school. With respect to religion, all of them were Muslims and their parity ranged from one to eleven. On the other hand, key informants were considered from diverse background and experience which included HEW (4), TBAs (6), community leaders (9), midwives (3), social mobilizer (2) and women and children affair office workers (7).

\section{Socio-cultural beliefs and traditions} influencing health facility birth: The study revealed that there were several socio-cultural beliefs and practices influencing childbirth at health facility which is organized into six overarching themes; namely, 1) preference and value for TBAs, 2) home delivery as intergenerational culture and suitable for privacy 3)culturally unacceptable birth practices and care at health facilities 4) inconvenience health facility for carrying out cultural ritual practices 5) social taboo regarding male involvement in delivery service and 6) fear and misperceptions of medical interventions.

Preference and value for TBA: In all study communities participants discussed that home delivery assisted by TBAs is the most valued and preferred care (Table 1). 
Table 1: Reasons for preference of TBAs to formal health workers, with illustrative quotes

\begin{tabular}{|c|c|}
\hline Reasons for preferences and narratives & Illustrative quotes \\
\hline \multirow{3}{*}{$\begin{array}{l}\text { Accumulated knowledge, experience, and } \\
\text { skill: participants discussed that TBAs are } \\
\text { older with significant experience whereas } \\
\text { health workers were regarded as young with } \\
\text { no substantial experience. }\end{array}$} & $\begin{array}{l}\text { Traditional birth attendants have good knowledge and } \\
\text { rich experiences. Some of them are better than health } \\
\text { workers (Community leader, Afar). }\end{array}$ \\
\hline & $\begin{array}{l}\text { When we go to the health facility, we could find only } \\
\text { young health workers. We are not happy to be touched by } \\
\text { young professionals. TBAs are better. } \\
\text { (Woman in FGD, BG) }\end{array}$ \\
\hline & $\begin{array}{l}\text {...only that little girl is a midwife and she didn't attend the } \\
\text { delivery, I and other three traditional birth attendants are } \\
\text { here in Obale [village]( Woman in FGD, Somali) }\end{array}$ \\
\hline \multirow{2}{*}{$\begin{array}{l}\text { Respect and ethics: Participants argued that } \\
\text { TBAs are humble and respectful, but the } \\
\text { health workers do not respect them and } \\
\text { perceived that they (health workers) } \\
\text { unnecessarily manipulate their genitalia } \\
\text { without their permission. They said that } \\
\text { touching of genitalia was a sign of disrespect } \\
\text { to women and against their culture. In Afar, } \\
\text { women preferred medical equipment instead } \\
\text { of hands to perform a vaginal examination. In } \\
\text { all communities, it was reported that TBAs } \\
\text { never insert a finger to the virginal canal for } \\
\text { examination. }\end{array}$} & $\begin{array}{l}\text { The young professionals abuse us; they rush to insert their } \\
\text { hand into the vagina.Milensah!...Milensah!...Milensah! } \\
\text { (never!...never!...never! We hate it! (Woman in FGD, BG) }\end{array}$ \\
\hline & $\begin{array}{l}\text { In the health center, we get no respect, they put their } \\
\text { finger into her body; they call it 'mermera '[examination]. } \\
\text { Afar women can't tolerate such disrespect. I haven't seen a } \\
\text { woman who praises the health center. (Woman in FGD, } \\
\text { Afar). }\end{array}$ \\
\hline $\begin{array}{l}\text { Privacy and confidentiality: Unlike health } \\
\text { workers who expose women's genital organ, } \\
\text { TBAs would perform all procedures } \\
\text { undercover without exposing the women's. } \\
\text { And she (TBA) also keep all private } \\
\text { information confidential and secrete. In BG } \\
\text { and Afar, participants also concerned that } \\
\text { health workers may also video record them } \\
\text { on camera. }\end{array}$ & $\begin{array}{l}\text { We have information that health workers use videos to } \\
\text { record women in labor; in fact, we know that there is no } \\
\text { privacy at all in a health facility; that is why we don't want } \\
\text { to give birth at a health facility (Woman in FGD, Afar). }\end{array}$ \\
\hline $\begin{array}{l}\text { Holistic and continuum care: Participants } \\
\text { expressed that TBAs provide care with } \\
\text { emotion, love, affection and psychological } \\
\text { support. }\end{array}$ & $\begin{array}{l}\text { (At home) They may call one of the family members to } \\
\text { hold the mother's back, in health facility they are afraid to } \\
\text { request such a help because people in a health facility are } \\
\text { not like their family, they are strange (HEW, BG). }\end{array}$ \\
\hline
\end{tabular}

Home delivery as inter-generational culture and as a place suitable for privacy: Most participants considered home as a natural place to give birth and considered it as a wonderful tradition passed down for generations.
'Our ancestors have been giving birth at their home. It is a wonderful tradition'

[Woman in FGD, Somali]

Participants in Afar and Somali communities explained that women should give birth in a traditional house which is featured with low

DOI: http://dx.doi.org/10.4314/ejhs.v29i3.6 
illumination that protects women's privacy. In contrast, it was reported that there is so much electric light in health facilities that expose their privacy by illuminating the room.

'At health institutions, they give birth in well-lit place while women need to give birth in dark traditional homes' [KII, Somali].

Culturally unacceptable birth practices at health facilities: All participants agreed that the delivery positions, being practiced in health facilities in which a woman was requested to lie on her back, was unacceptable to women, and generally against their cultural norms and tradition. They believed that the position extremely exposes a woman's privacy. Participants from Somali and BG reported that, according to their tradition, women give birth at home on kneeling position.

'...at health facility women lay on their back to give birth; they hate that very much because their naked body is exposed to people; that is unacceptable in our religion' [Elderly woman, BG].

In Afar, participants worried that health workers let women in labour lie on their side which they perceived can bend the fetus and make the birth more difficult. In this community, women prefer sitting position to give delivery.

'We also heard that health workers order her to lie on the side that can cause a fetus to bend [Women in FGD, Afar].

All women in the FGDs and most KIIs strongly objected to the use of delivery coach because they believed that it further exacerbates the invasions of women's privacy.

'They feel shy and fearful to lie on the delivery coach. They usually say that they don't want to go up on the delivery coach and open legs wildly' [midwifery, Somali].

Lack of support during labour: Participants were concerned that unlike TBAs, health workers do not encourage and support a woman to push the baby out.

...TBA massage her back and abdomen with butter, her mother or other family members support her from the back in a sitting position. We give her camel's milk [KII-Afar].
Objection to be assisted by male health workers: Participants explicitly stated that provision of delivery service by male health worker is generally unacceptable; against their culture and religious belief.

'... They [women] would prefer to die at home than be seen by men. There is a care in it but prefer to die at home than seen by men' [Woman in FGD, Afar].

Since we are Muslims; our religion and culture does not allow male attendance... [Woman in FGD, BG].

Misperceptions and fear of medical interventions: Participants in BG and Somali worried that health workers tended to incise women's genital organ with scissors without their consent. In connection with this, women experienced verbal and physical abuses from health workers.

We have fear because health workers cut woman's genital organ with scissors and pinch them while they are in labor. They say don't cry! don't disturb us! It is not your home! Due to this problem, none of the women will go to the health facility to give birth [Woman in FGD, BG].

Several participants argued that TBAs do not use scissors to cut off women's genital organ.

...but our birth attendant knows, she can rotate the head of the baby and manage it' [Woman in $F G D, B G]$.

Women in Afar prefer vaginal incision or episiotomy. Unlike in Somali and BG regions, TBAs in Afar use a knife to cut or tear the vagina. Participants in this region said that women do not like to visit a health facility for delivery since no such service is given by health workers.

We have been told that health workers don't use a knife and let her [woman] deliver by herself just like goats which deliver by themselves. We don't like to strain like goats! We are not goats![Woman in FGD, Afar].

Health facilities are inconvenient for cultural ritual practices: There was consensus on the fact that childbirth needs to be accompanied by the performance of several cultural rituals. 
Participants ascertained that health facility is not a suitable place to perform cultural rituals during labor and delivery.

If we deliver at the health facility, we couldn't 'get Almahaya'. If we deliver at the health facility our Sheik will never do that! That is why we hate to deliver at a health facility[Woman in $F G D, B G]$.

Participants mentioned that it was impossible for them to provide emotional support, and care women need from families and health staffs do not allow TBAs to stay with a laboring woman in delivery rooms.

They take a woman and close their door. They leave TBA outside.)[KII TBA, in Afar].

There is a general belief that the newborn should get a bath, massage with butter and some local materials should be put on the baby's head to protect him/her from an evil spirit. In addition, the newborn is fed with butter, porridge, oil, milk, sugar, and tea immediately after birth, especially when the mother's milk is unavailable or insufficient. Participants stated that all these practices were not allowed in health facilities.

Azan, Islamic call, and ceremony: In all study communities, participants explained that the newborn should hear 'Azan"2 up on birth. According to them, the newborn should hear the Azan before feeding anything. In Afar, they have a special ceremony called 'Onqor' or 'Unori' which extends the time of breastfeeding initiation further. Onqor takes place the next morning facing the sunrise. The newborn should not breastfeed before the ceremony. For a baby girl, onqor can be performed by TBA or any woman, but for baby boy, it is usually performed by the elderly or sheik. They complained that health facilities are not suitable to perform Onqor.

If a baby boy is born at night, they will give him nothing even breast milk before "onqor". Onqor is practiced in the next morning facing towards the morning sun. For a baby girl, no man is invited to perform Onqor; if it is in the night the

1 a glass of water over which a Sheik reads from Qur'an and pray on it to relieve pain

2 "Azan" is the Muslim call to ritual prayer made by a muezzin from the minaret of a mosque
TBA stays the night with the baby girl and practices 'onqor' the next morning[HEW, Afar].

Culturally accepted hygiene practice: Respondents reported that health facility setting was not convenient to keep mother's and newborn's hygiene as per their cultural values and standards.

'When a woman gives birth at home, a small hole is dug in her room in order to discard her and her baby's waste like blood, feces, and urine. In our culture, even the baby's dirty water after washing should never be discarded outside the room until 15 days. It should be buried in the prepared hole to prevent the baby and the mother from Shetan. Even their clothes should never be put outside. We dislike giving birth at a health facility because health facilities do not allow such practices [Woman in FGD, BG].

Culturally acceptable food preference and availability: Participants mentioned that health facilities are not convenient to prepare cultural foods and drinks which should be given to a woman while she is in the health facility. In their opinions, camels' milk, tea, honey, and sugar dissolved in water help the mother to push the baby strongly and shorten recovery time. Butter and meat were also mentioned as good foods after delivery as it is believed to enhance bleeding thereby reducing blood clotting in the womb.

We give her (the mother) hot butter to prevent blood clotting. We don't prevent bleeding after the baby comes out; we consider it as healthy process; if blood clotted and is retained in the womb, it leads to severe pain and death [Opinion leader, Somali].

When the delivery is at home, they perform different practices to reduce some problems women would be facing such as fastening/tightening the woman's abdomen and making her lie down without pillow (to reduce bleeding), closing/tightening thighs (to reduce further bleeding), inserting salt to a woman's anus and giving meat taken from tail of sheep (to alleviate abdominal pain), and home confinement (to avoid evil spirits).

${ }^{3}$ Satan

DOI: http://dx.doi.org/10.4314/ejhs.v29i3.6 
.... We tighten her abdomen, let her lie down without pillow, close her thighs, to reduce bleeding. We rub her head with butter in our rural ritual (tradition) [Woman in FGD, Somali].

\section{DISCUSSION}

This study explored diverse socio-cultural beliefs and practices influencing institutional delivery service utilization in three communities of Ethiopia. The positive value and the high preference laid on TBAs was strongly linked to avoidance of health facility birth. Women have full confidence in the competence and ability of TBAs in assisting delivery. In contrast, women do not trust and are worried about the skills, experiences, and competence of health workers. Earlier studies also documented similar perceptions (11-17).

In Ethiopia, there has been an effort to discourage the role of TBAs in delivery assistance. Nevertheless, the value and preference for TBAs have remained very strong in the study communities. This suggests the need to revisit the value and potential role of TBAs in delivery assistance especially in such communities which have a strong value for TBAs. Besides preferring TBA to health professionals, the community perceive home delivery as inter-generational culture, suitable for privacy and perceived as a natural place to give birth.

The delivery service approaches being practiced at health facilities such as the use of a delivery coach and inserting fingers to the vagina for examination is culturally unacceptable to women and community members. The fact that health workers often perform physical examination without obtaining informed consent exacerbates the problem. The birth position (i.e. let the woman lie on her back, bend her legs and open her knees) is incompatible with the community's cultural norms. On top of this, physical and verbal abuse (hitting or roughly forcing legs apart), non-dignified care (such as humiliation by shouting, or degrading) and nonconsented care (e.g. performing examination without consent) contributed to a women's decision to avoid health facility birth. This is in line with some earlier studies $(13,18-20)$.
Inconsistent with some previous studies $(21,22)$, the study has shown that women value emotional and psychological support from family members, relatives, and TBAs during labour and the postpartum period. Indeed, continuous emotional and psychological support during childbirth has clinically meaningful benefits for women and newborns (22). Consequently, women in these communities tend to avoid health facility birth due to the absence of these supports at health facilities.

Fear of medical interventions mainly episiotomy was found to be one of the reasons for women to avoid health facility birth. In fact, the perceptions were different between communities. In BG and Somali regions, women are against episiotomy procedures, whereas in Afar, women generally avoid health facility birth perceiving that no episiotomy or deinfibulation of vaginal opening service at the health facility. Thus, it is important to have a discussion regarding deinfibulations and infibulations early during antenatal care visits.

The study also found that there were multiple cultural rituals like the offering of special cultural foods and drinks for the newborn and the women in labour, hygiene practices, praying and blessings during childbirth. Moreover, there was a belief that the woman and the newborn should take bath in order to promote hygiene, healing, and recovery, and to prevent some health problems. In addition, handling and disposing of the placenta has culturally accepted procedures, and health workers need to understand the cultural context of handling and disposing placenta and educate families as appropriate.

On the other hand, not being able to conduct 'Azan' to a newborn baby by a religious leader, was found to be a key obstacle to institutional delivery service uptake. This ritual needs to be acknowledged and addressed in delivery care through involving religious leader. Nevertheless, the 'Onqor' ceremony in Afar communities which may cause a significant delay in initiating breastfeeding needs urgent educational intervention to abandon it.

Another factor which greatly influenced the use of delivery service was delivery service by 
male health workers. Some studies elsewhere also reported similar beliefs $(12,14)$. Hence, it is important to review the profile of health workers deployed in the area to provide delivery services and take appropriate actions such as training (inservice and basic) of additional female health workers. Simultaneously, it is also important to initiate dialogue with community members on this issue.

This study underscored that understanding socio-cultural norms, traditions and beliefs on childbirth is a first step in developing interventions that promote skilled birth attendances. It identified similarities and contrasts between the study communities which could help for localized actions. However, even though purposeful sampling was appropriate for in-depth exploration of participants' perspectives on the issue, the small sample size and the focus on three communities may limit transferability of the results. This requires effectively mapping the socio-cultural factors affecting institutional delivery in the country in order to adapt culturally appropriate birthing services.

In conclusions, the study indicated that there were diverse socio-cultural beliefs and practices which greatly influence women's' decision to use health facilities for childbirth. TBAs remain the major choice for delivery assistance in the study communities even when skilled health professionals exist and are accessible to the communities; calling for the need to revisit the potential of TBAs for improved birth outcomes. On the other hand, the conventional delivery assistance procedures being practiced at health facilities were failed to consider communities' cultural and rituals needs which ultimately led to the rejection of health facility birth. Use of male health workers in delivery care and misperception around the use of medical interventions had substantial impacts on seeking delivery care. Thus, in order to promote institutional delivery services in the study communities, it is essential to re-orient health facility birth to provide culturally competent care that fits women's need, and the community's socio-cultural context. It is also vital to adopt beneficial norms and establish a women-friendly, home-like birth environment in health facilities to attract women for birth.

\section{ACKNOWLEDGMENTS}

We thank study participants, regional and local administrators, health staffs, and community members who participated in the study and facilitated field work. We also acknowledge the data collection team. Finally, we express our heartfelt thanks to the Ethiopian Public Health Institute for financing to carry out this study.

\section{REFERENCES}

1. WHO. Maternal mortality: WHO fact sheet on maternal mortality with key facts and providing information on MDG 4, where deaths occur, causes, lack of care and WHO response. World Health Organization. 2018 [cited 2018 Nov 8]. Available from: http://www.who.int/news-room/factsheets/detail/maternal-mortality

2. World Health Organization, UNICEF, UNFPA, The World Bank \& the United Nations Population Division. Maternal mortality in 1990-2013: Ethiopia. Geneva: WHO; 2014.

3. Ethiopia Central Statistical Agency and ICF International. Ethiopia Demographic and Health Survey: Key Findings [Internet]. Central Statistical Agency Addis Ababa, Ethiopia; 2016 [cited 2018 Oct 9]. Available from: https://dhsprogram.com/publications/public ation-fr328-dhs-final-reports.cfm

4. Ronsmans C, Graham WJ. Maternal mortality: who, when, where, and why. The Lancet [Internet]. 2006 Sep 30 [cited 2018 Nov 8];368(9542):1189-200. Available from:

https:/www.thelancet.com/journals/lancet/a rticle/PIIS0140-6736(06)69380-X/abstract

5. WHO | Countdown to 2015: taking stock of maternal, newborn \& child survival [Internet]. [cited 2018 Nov 8]. Available from:

http://www.who.int/maternal_child_adolesc ent/documents/9789241599573/en/

DOI: http://dx.doi.org/10.4314/ejhs.v29i3.6 
6. Graham WJ, Bell JS, Bullough CHW. Can skilled attendance at delivery reduce maternal mortality in developing countries? Safe Mother Strateg Rev Evid Eds BrouwereVVan LerbergheW Stud Health Serv Organ Policy [Internet]. 2001 [cited 2018 Nov 8]; Available from: https://abdn.pure.elsevier.com/en/publicatio ns/can-skilled-attendance-at-deliveryreduce-maternal-mortality-in-d

7. Ethiopia Central Statistical Agency and ICF International. Ethiopia Demographic and Health Survey: Key Findings [Internet]. Central Statistical Agency Addis Ababa, Ethiopia; 2011 [cited 2018 Oct 9]. Available from: https://dhsprogram.com/publications/public ation-fr255-dhs-final-reports.cfm

8. Gabrysch S, Campbell OM. Still too far to walk: Literature review of the determinants of delivery service use. BMC Pregnancy Childbirth [Internet]. 2009 Aug 11 [cited 2018 Nov 8];9(1):34. Available from: https://doi.org/10.1186/1471-2393-9-34

9. IRIN. Still too many deaths in childbirth [Internet]. [cited 2018 Nov 8]. Available from:

http://www.irinnews.org/news/2012/04/25/s till-too-many-deaths-childbirth

10. Barney Glaser, Anselm Strauss. The Discovery of Grounded Theory: Strategies for Qualitative Research [Internet]. [cited 2018 Nov 8]. Available from: https://www.amazon.com/DiscoveryGrounded-Theory-StrategiesQualitative/dp/0202302601

11. Shiferaw S, Spigt M, Godefrooij M, Melkamu Y, Tekie M. Why do women prefer home births in Ethiopia? $B M C$ Pregnancy Childbirth [Internet]. 2013 Dec [cited 2018 Nov 8];13(1):5. Available from: https://bmcpregnancychildbirth.biomedcentr al.com/articles/10.1186/1471-2393-13-5

12. Sialubanje C, Massar K, Hamer DH, Ruiter RA. Reasons for home delivery and the use of traditional birth attendants in rural Zambia: a qualitative study. $B M C$ Pregnancy Childbirth [Internet]. 2015 Dec [cited 2018 Nov 8];15(1):216. Available from:

https://bmcpregnancychildbirth.biomedcentr al.com/articles/10.1186/s12884-015-0652-7

13. Caulfield T, Onyo P, Byrne A, Nduba J, Nyagero J, Morgan A, et al. Factors influencing place of delivery for pastoralist women in Kenya: a qualitative study. BMC Women's Health [Internet]. 2016 Aug 9 [cited 2018 Nov 8];16. Available from: https://www.ncbi.nlm.nih.gov/pmc/articles/ PMC4979142/

14. al SB et. Reasons for Preference of Home Delivery with Traditional Birth Attendants (TBAs) in Rural Bangladesh: A Qualitative Exploration. - PubMed - NCBI [Internet]. [cited 2018 Nov 8]. Available from: https://www.ncbi.nlm.nih.gov/pubmed/2673 1276

15. Kaba M, Bulto T, Tafesse Z, Lingerh W, Ali I. Sociocultural determinants of home delivery in Ethiopia: a qualitative study. Int $J$ Womens Health. 2016;8:93-102.

16. Temesgen TM, Umer JY, Buda DS, Haregu TN. Contribution of traditional birth attendants to the formal health system in Ethiopia: the case of Afar region. Pan Afr Med J [Internet]. 2012 Dec 26 [cited 2018 Nov 8];13(Suppl 1). Available from: https://www.ncbi.nlm.nih.gov/pmc/articles/ PMC3587022/

17. Balde MD, Bangoura A, Diallo BA, Sall O, Balde $H$, Niakate AS, et al. A qualitative study of women's and health providers' attitudes and acceptability of mistreatment during childbirth in health facilities in Guinea. Reprod Health [Internet]. 2017 Jan 13 [cited 2018 Nov 8];14(1):4. Available from: https://doi.org/10.1186/s12978-0160262-5

18. Mehretie Adinew $\mathrm{Y}$, Abera Assefa N. Experience of Facility-Based Childbirth in Rural Ethiopia: An Exploratory Study of Women's Perspective [Internet]. Journal of Pregnancy. 2017 [cited 2018 Nov 8]. Available from: https://www.hindawi.com/journals/jp/2017/ 7938371/

19. An ethnographic account of the beliefs, values, and experiences of rural Swazi

DOI: http://dx.doi.org/10.4314/ejhs.v29i3.6 
women during childbirth | RCM [Internet]. [cited 2018 Nov 8]. Available from: https://www.rcm.org.uk/learning-andcareer/learning-and-research/ebmarticles/an-ethnographic-account-of-thebeliefs-values

20. Miller S, Lalonde A. The global epidemic of abuse and disrespect during childbirth: History, evidence, interventions, and FIGO's mother-baby friendly birthing facilities initiative. Int $J$ Gynecol Obstet [Internet]. 2015 Oct 1 [cited 2018 Nov 8];131:S49-52. Available from: http://www.sciencedirect.com/science/articl e/pii/S0020729215000843.
21. Aziato L, Odai PNA, Omenyo CN. Religious beliefs and practices in pregnancy and labour: an inductive qualitative study among post-partum women in Ghana. $B M C$ Pregnancy Childbirth [Internet]. 2016 Jun 6 [cited 2018 Nov 8];16(1):138. Available from: https://doi.org/10.1186/s12884-0160920-1

22. Hodnett ED, Gates S, Hofmeyr GJ, Sakala $\mathrm{C}$, Weston J. Continuous support for women during childbirth. Cochrane Database Syst Rev. 2011 Feb 16;(2):CD003766. 